\title{
Clinical Utility Of Elagolix As An Oral Treatment For Women With Uterine Fibroids: A Short Report On The Emerging Efficacy Data
}

This article was published in the following Dove Press journal: International Journal of Women's Health

\author{
Manuela Neri $\mathbb{D}^{1-3}$ \\ Gian Benedetto Melis (DD ${ }^{1-3}$ \\ Elena Giancane ${ }^{1-3}$ \\ Valerio Vallerino ${ }^{1-3}$ \\ Monica Pilloni $\mathbb{D}^{1-3}$ \\ Bruno Piras (ID) ${ }^{1-3}$ \\ Alessandro Loddo 1-3 \\ Anna Maria Paoletti (D) ${ }^{1-3}$ \\ Valerio Mais ${ }^{1-3}$ \\ 'Department of Obstetrics and \\ Gynaecology, University of Cagliari, Italy; \\ ${ }^{2}$ Department of Surgical Sciences, \\ University of Cagliari, Italy; ${ }^{3}$ Maternal \\ Child Department, University Hospital of \\ Cagliari, Italy
}

\begin{abstract}
Uterine fibroids (UFs) are the most common gynaecological benign disease. Even though often asymptomatic, UFs can worsen women's health and their quality of life, causing heavy bleeding and anaemia, pelvic discomfort and reduced fertility. Surgical treatment of UFs could be limited by its invasiveness and the desire to preserve fertility. Thus, effective medical therapies for the management of this condition are needed. Common drugs used to control bleeding, such us hormonal contraceptive or levonorgestrel-releasing intrauterine system, have no effect on fibroids volume. Among other more efficient treatments, the gonadotropin-releasing hormone $(\mathrm{GnRH})$ agonist or the selective progesteronereceptor modulators have a non-neutral safety profile; thus, they are used for limited periods or for cyclic treatments. Elagolix is a potent, orally bioavailable, non-peptide GnRH antagonist that acts by a competitive block of the GnRH receptor. The biological effect is a dosedependent inhibition of gonadal axis, without a total suppression of estradiol concentrations. For this reason, even though comparative studies between elagolix and $\mathrm{GnRH}$ agonists have not been performed, elagolix has been associated with a better profile of adverse events. Recently, elagolix received US FDA approval for the treatment of moderate to severe pain caused by endometriosis. Several clinical trials assessed the efficacy of elagolix for the treatment of heavy bleeding caused by UFs and the definitive results of Phase III studies are expected. Available data on elagolix and UFs showed that the drug, with or without low-dose hormone add-back therapy, is able to significantly reduce menstrual blood loss, lead to amenorrhea and improve haemoglobin concentrations in the majority of participants in comparison with placebo. The safety and tolerability profile appeared generally acceptable. The concomitant use of add-back therapy can prevent bone loss due to the hypoestrogenic effect and can improve safety during elagolix treatment.
\end{abstract}

Keywords: uterine fibroids, leiomyomas, elagolix, non-peptide GnRH antagonist, heavy menstrual bleeding

\section{Introduction}

Uterine fibroids (UFs), also known as uterine myomas or leiomyomas, are the most frequent gynaecological benign disease. Establishing the real prevalence of this condition is complex because, very often, they develop without any sign or symptoms. A recent epidemiological study estimated that the prevalence of UFs reaches the $70 \%$ of women, with an age-dependent increase of the incidence. ${ }^{1}$ The black race is considered the main risk factor, as it increases UFs appearance by twothreefold compared with the white race. Among other secondary risk factors,
Correspondence: Manuela Neri Department of Obstetrics and Gynaecology, Department of Surgical Sciences, University of Cagliari, Maternal Child Department, University Hospital of Cagliari, Policlinico Universitario Duilio Casula, SS 554 - bivio Sestu, blocco Q Monserrato, Cagliari 09042, Italy Tel +393338867567

Email manu.nerilI@hotmail.it 
conditions associated with increased ovarian steroids exposure, such as early menarche, nulliparity or first pregnancy delayed in late age, are to be included. ${ }^{1-3}$ Despite the high prevalence, UFs become symptomatic, sometimes seriously, in only $15-30 \%$ of cases. ${ }^{2}$ Symptoms linked to myomas can depend on their localization in the uterus: submucosal fibroids are more likely related to heavy menstrual bleeding (HMB) and anaemia, whereas subserosal fibroids can cause compressive symptoms such as pain, dysuria, tenesmus or constipation. ${ }^{2}$ Not surprisingly, UFs may also affect reproduction and fertility in different ways: by altering the endometrial cavity and its perfusion, by reducing implantation or by affecting endocrine and paracrine molecular mechanisms involved in embryonic development. ${ }^{4-6}$ Moreover, the presence of fibroids has been linked to adverse pregnancy outcomes, such as increased risk of spontaneous abortion, foetal malpresentation, placenta previa, preterm birth, caesarean section, and peripartum haemorrhage. ${ }^{7}$ Last but not least, medical literature reports a lot of evidence that UFs and related symptoms negatively affect women's quality of life (QoL). Cross-national surveys demonstrated that more than $60 \%$ of women with leiomyomas present a moderate to very important impact of fibroids on quality of life, ${ }^{8}$ and work productivity and general activity are reduced by more than 35\%. ${ }^{9}$ Common UFs symptoms affecting health-related QoL are lower back pain (65\%), fatigue (63\%), bloating (61\%), pelvic pain/cramping during menses (63\%), and HMB (54\%). ${ }^{10}$ As a consequence of the great prevalence, the chronicity of the condition, the burden of the symptoms and the impact on daily life, UFs imply a considerable cost for both patients and health care systems, which has been estimated to be greater than that of some malign diseases such as ovarian or breast cancer. ${ }^{11}$ For this reason, medical research has invested and is investing heavily in the development of efficient treatment for the UFs. There is a general consensus not to treat myomas when they do not cause symptoms. In all other case, the treatment of UFs can take advantage of surgery, medical therapies or physical treatments, and each approach presents different pros and cons. The management must consider clinical manifestation, leiomyomas features (number, size and site), and patient's preference relating for example to pregnancy desire. ${ }^{12}$

\section{Surgical And Physical Treatments Of UFs}

A surgical approach is the only one that allows a radical treatment of fibroids. Although hysterectomy definitely solves the problem of UFs and their recurrence risk, ${ }^{12}$ for its invasiveness it should be considered in symptomatic women that do not wish to give birth and agree with a surgical approach. ${ }^{13}$ To preserve fertility, conservative treatment such as myomectomy could be considered. In this case, the laparoscopic approach showed a more favorable fertility rate after surgery compared to the abdominal, with lower morbidity and shorter hospitalization after surgery. ${ }^{14}$ However, when myomas are numerous, large or deep in the myometrium, laparoscopy becomes more difficult and may require the use of morcellation or minilaparotomy to remove the lesions. ${ }^{13,15}$ As a small, but not irrelevant, risk of disseminating an occult sarcoma exists during fibroids extraction procedures, the Food and Drug Administration warns against using morcellation. ${ }^{16}$ Containment systems like endoscopic bags are now available for both electrical and manual morcellation. ${ }^{17}$ For sub-mucous fibroids, hysteroscopic myomectomy is the treatment of choice to improve bleeding and to normalize the uterine cavity. ${ }^{18}$ In selective cases of UFs associated to metrorrhagia, in women who do not wish to preserve fertility, a treatment option could be also hysteroscopic endometrial ablation. ${ }^{12,13}$ In different contexts, such as strongly symptomatic and voluminous myomas, surgical treatment of UFs represents the gold standard. Nevertheless, alternatives to surgery are necessary because of its invasiveness, its cost and its reproductive consequences. Last but not least, it must be considered that many women often prefer not to undergo surgery. ${ }^{19}$ Lately, non-surgical physical treatments for UFs have been developed. The uterine artery embolization is a percutaneous catheterization technique that causes a selective necrosis of the fibroids, preserving the healthy myometrium that has the capacity to revascularize itself. This minimally invasive procedure has the advantages that it can act at the same time on several lesions and it is associated with short hospitalization and rapid recovery times. On the other hand, the procedure causes sometimes severe abdominal pain and has the risk of pelvic infection or incomplete infarction that requires a further intervention. Moreover, the effects on reproductive outcomes are not defined, so it cannot be proposed to women seeking pregnancy. ${ }^{20}$ Another innovative minimally invasive technique is high-intensity focused ultrasound fibroids ablation. It uses ultrasound energy, with the help of magnetic resonance or ultrasound to see the lesions, and causes focal coagulation and necrosis of fibroids. As this technique is new and still not much used, evidence is needed to prove 
its efficacy and its safety. ${ }^{13,20}$ Great interest is arising from myomas radiofrequency ablation, which is a new and innovative minimally invasive technology applied to surgery: it consists in the use of an electrode that induces hyperthermic necrosis of fibroids, sparing the healthy uterine tissue surrounding, during laparoscopic procedure or, very recently, by transcervical route, under ultrasound guide. $^{21,22}$ This technique is effective in treating UFs of various sizes and number, even if the best results are obtained on less bulky fibroids, it presents rapid recovery, low postoperative pain and good improvement of QoL; the long-term effects on pregnancy outcomes are still little known. $^{21}$

\section{Medical Treatments Of UFs}

Uterine fibroids derive from the proliferation and the growth of smooth-muscle cells of the myometrium. ${ }^{2}$

In vitro studies hypothesize that myomas originate from a single-mutated myometrial stem cell that acquires the capacity to proliferate, differentiate into smooth-muscle cells and produce extracellular matrix..$^{23,24}$ Different endocrine and paracrine factors can be involved in myomas growth, with ovarian steroids that play a central role: fibroid tissue presents both estrogen receptors (ER) $\alpha$ and progesterone receptors (PR); estrogens promote the expression of PR, while progesterone is the main factor that stimulates fibroid cell hypertrophy and hyperplasia. ${ }^{25,26}$ Based on this evidence, medical treatments of UFs generally aim to remove the hormonal stimulation of myomas cells. Drugs commonly used can improve symptoms related to UFs and reduce the growth, but they are not able to eradicate the lesions. A medical treatment could be considered in women who refuse a surgical approach or as a first-line treatment in women near to menopause. Nevertheless, the onset of adverse events often limits the use of prolonged pharmacological therapies.

Considering that leiomyoma cells express more aromatase than normal myometrium, ${ }^{27}$ aromatase inhibitors have been tested in the UFs treatment. There is some evidence that these drugs can reduce fibroid size and improve associated symptoms. ${ }^{28,29}$ Nevertheless, a long-term treatment can be limited by the adverse effects of prolonged hypoestrogenism and there is no strong evidence to recommend aromatase inhibitors for UFs. ${ }^{30}$

Combined oral contraceptives are sometimes used to control bleeding in patients with fibroids, even if no valid data exist that support an efficacy on myomas growth and volume. $^{31}$ Levonorgestrel-releasing intrauterine systems (LNG-IUS) demonstrated to markedly reduce menstrual bleeding in women with symptomatic fibroids. ${ }^{32}$ The ability of LNG-IUS to down-regulate PR expression in the uterus $^{33}$ could decrease fibroid receptivity to ovarian hormone stimulation. However, changes in uterine and fibroid volumes are inconsistent during LNG-IUS therapy. ${ }^{32}$

In view of antiproliferative, anticancer, immunomodulant and antioxidant properties, some substances such as vitamin D, curcumin and green tea extract could exert a favourable effect against the UFs growth. Moreover, various epidemiological studies reported a vitamin D deficiency as a risk factor for the development of myomas. Consequently, these alternative medicaments are under investigation for their possible role in the prevention and the treatment of UFs. ${ }^{34,35}$

Among the drugs registered for UFs treatments, gonadotropin-releasing hormone agonists (GnRHa) are the oldest and best-known drugs approved to treat symptoms related to leiomyomas. They act by suppressing ovarian steroids secretion; thus, GnRHa have efficacy in reducing fibroid-related bleeding and anemia, uterine and fibroid size. ${ }^{20}$ Unfortunately, after treatment discontinuation, fibroids regrowth and recurrence of symptoms invariably reappear. ${ }^{36}$ Furthermore, the frequent hypoestrogenic adverse events (hot flushes, insomnia, mood disorders, vaginal dryness) and the risk of bone mineral density loss hinder long-term therapy with GnRHa, so these drugs can be used safely for a short time, ${ }^{37}$ for example as preoperative treatment, or with hormonal add-back therapy to reduce adverse events. A 3month treatment with GnRHa before surgery showed in a systematic review to significantly improve both preoperative and postoperative hemoglobin levels, and to significantly reduce operative time and hospital stay. ${ }^{38}$ The use of an add-back therapy can mitigate adverse events related to prolonged use of GnRHa: there is a modest evidence that tibolone, raloxifene, estriol and ipriflavone can help in reducing bone loss and that medroxyprogesterone acetate (MPA) and tibolone may antagonize moderate vasomotor symptoms, even if some add-back therapies can limit the effect of GnRHa in reducing uterine volume. ${ }^{39}$ Consequently, in clinical practice, GnRHa is used for no more than 6 months.

A more recent therapy studied for symptomatic UFs is that of selective progesterone receptor modulators (SPRMs). Binding PR, the SPRM can exert both agonism and antagonism, with a biological effect that depends on the type of PR expressed and the affinity and strength of bond of each modulator. ${ }^{40}$ Concerning uterine fibromatosis, in laboratory studies SPRMs demonstrated to exert antiproliferative, proapoptotic, and antifibrotic changes selectively 
on myoma cells, in addition to the reduction of pituitary gonadotropin secretion that can lead to amenorrhea. ${ }^{41}$ To date, ulipristal acetate (UPA) is the only SPRMs approved as medical therapy of UFs in Europe and Canada. In premarketing trials, UPA showed efficacy in reducing HMB, fibroids size and in improving hemoglobin blood concentration and QoL; ${ }^{42}$ bleeding control is achieved more quickly than with $\mathrm{GnRHa}^{43}$ and tolerability with UPA can be better than with GnRHa because the SPRM does not exert a complete estrogen suppression. ${ }^{44}$ Initially, UPA has been recommended for 3-month preoperative treatment in women with HMB and UFs. Recent evidence supported the long-term intermittent UPA treatment (repetitive cycles of 3 months, alternated with 2 menstrual periods), is able to improve the effect on uterine size and to maintain a good health profiles. ${ }^{45,46}$ In view of the widespread use of UPA, some rare reports of serious liver injury, including liver failure, recently emerged, thus the European Medicines Agency (EMA) Pharmacovigilance Risk Assessment Committee (PRAC) carried out a full evaluation about UPA safety. The PRAC concluded that UPA may have contributed to the development of some cases of serious liver injury and recommended measures to minimize liver injury risk (such as frequent liver tests during treatment), restricting the indication for one course (up to 3 months) of preoperative treatment for moderate-severe symptomatic UFs and for prolonged intermittent use only in women who are not eligible for surgical treatment. ${ }^{47}$ Other SPRMs, such as vilaprisan, are now under evaluation in Phases II and III studies, for the treatment of HMB associated with UFs. ${ }^{48}$

Therefore, to date, there are no medical therapies available for the chronic treatment of symptomatic UFs that are effective, safe and with good tolerability. Thus, clinical research is investing heavily in this field. The novel gonadotropin-releasing hormone antagonists (GnRHant) are promising drugs for prolonged treatment of different gynecological conditions, including uterine fibromatosis.

\section{Gonadotropin-Releasing Hormone Antagonists}

The oldest GnRHant, like cetrorelix or ganirelix, are peptide molecules available only for injective use, usually used in the assisted reproductive medicine protocol. There is some evidence about their action in the treatment of UFs: cetrorelix demonstrated to be efficacious and well tolerated as preoperative treatment of fibroids; ${ }^{49}$ ganirelix treatment showed to induce a significant reduction of fibroid size in a median of 19 days. ${ }^{50}$ Compared to the agonists, the GnRHant could be advantageous because they immediately reduce the gonadotropin secretion, avoiding the initial stimulatory phase of the agonists. However, the peptide GnRHant have been associated with a significant risk of histamine-related adverse events and hypersensitivity reactions. ${ }^{51}$ Consequently, over the last 15-20 years, scientific research has worked towards developing of new molecules, available also for oral administration, with the aim of improving dose flexibility and patient acceptance. Now, different pre-clinical and clinical studies are in progress testing several non-peptide GnRHant. ${ }^{52}$ For the treatment of UFs, only 3 molecules have reached evaluation in Phase II and III clinical trials: elagolix, relugolix or TAK-385 and linzagolix or OBE-2109. Phase III study on relugolix for the treatment of HMB dependent on UFs are still ongoing, such as studies evaluating the efficacy of the drug for the treatment of endometriosis-associated pain and prostate cancer. In phase III trials, relugolix demonstrated a noninferiority compared to leuprorelin in terms of improvement of HMB after 6-12 weeks of treatment, with a more rapid onset of effect. Recently, relugolix (Relumina ${ }^{\circledR}$, Takeda Pharma, Tokyo, Japan) received its first global approval in January 2019 for marketing in Japan as a treatment for symptomatic uterine fibromatosis. ${ }^{53}$ OBE-2019 is now under evaluation compared to placebo in Phase III trials in women with HMB related to UFs, ${ }^{54,55}$ and in a Phase II trial for the treatment of pain associated with endometriosis. ${ }^{56}$

\section{Elagolix}

Elagolix is the most studied GnRHant in human clinical trials. Developed by AbbVie in collaboration with Neuroendocrine Biosciences, elagolix is a potent orally active non-peptide GnRHant. As chemical compound, it is an uracil derivative, in particular, an uracil-phenyl-ethylamine bearing a butyric acid. The specific chemical formula and the structure of elagolix are shown in Figure 1. In July 2018, the US FDA approved elagolix tablets for the treatment of moderate to severe pain associated with endometriosis, at the recommended dose of $150 \mathrm{mg}$ once daily for up 24 months, or, in patients with coexisting dyspareunia, at the dose of $200 \mathrm{mg}$ twice daily for up 6 months. ${ }^{57}$ The approval was obtained thanks to the positive results of the Phase II and especially Phase III trials conducted worldwide in patients with endometriosis-associated pain. ${ }^{58,59}$ Further Phase III trials for the same gynecological condition are currently ongoing evaluating this GnRHant with or without low-dose hormone add-back 


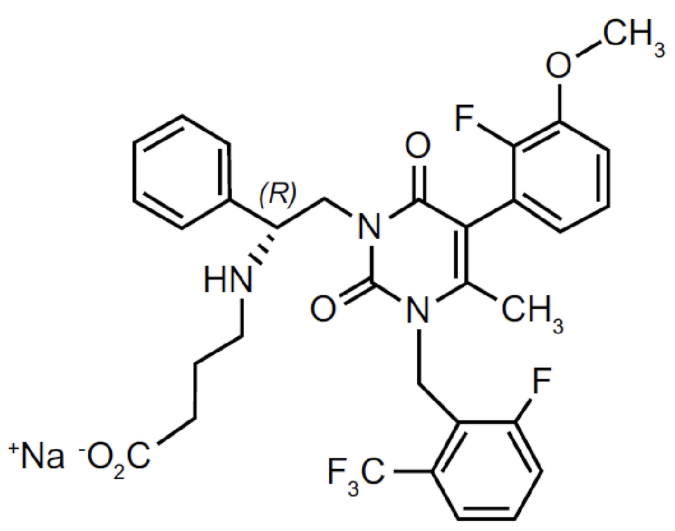

R-4-\{2-[5-(2-fluoro-3-methoxyphenyl)-3-(2-fluoro-6-[trifluoromethyl]benzyl)-4-methyl2,6-dioxo-3,6-dihydro-2H-pyrimidin-1-yl]-1-phenylethylamino\} butyric acid sodium salt

Figure I Chemical structure and molecular formula of elagolix.

therapy. ${ }^{57}$ Moreover, as to be expected, elagolix has generated great interest as therapy for uterine leiomyomas and related $\mathrm{HMB}$, and in this context, the drug is still under evaluation with encouraging results.

\section{Pharmacokinetics And Pharmacodynamics}

Elagolix acts as a highly potent antagonist of the human $\mathrm{GnRH}$ receptors. In pre-clinical study in castrated macaques, the oral administration of elagolix suppressed LH levels; at the oral doses of $30 \mathrm{mg} / \mathrm{kg}$, the inhibitory effect on LH secretion resulted more potent and rapid in comparison to other non-peptide GnRH antagonists. ${ }^{60}$ The pharmacodynamic data from the first Phase I study, conducted in premenopausal healthy female subjects, showed that a single $25 \mathrm{mg}$ dose of elagolix is able to quickly reduce $\mathrm{LH}$ and FSH concentrations; higher doses lead to a longer gonadotropin suppression, even though, after a single dose, baseline gonadotropin levels return to basal values within 24 hrs. $^{61}$ Mean estradiol concentration declines with at least $50 \mathrm{mg}$ single dose treatment and estradiol levels remain partially suppressed after $24 \mathrm{~h} ; 100 \mathrm{mg}$ twice daily showed a consistent significant suppression of estradiol levels compared with placebo. Daily 50-200 mg or twice daily $100 \mathrm{mg}$ of elagolix for 7 days during the midfollicular phase in most women prevents the estradiol midcycle-peak. After the discontinuation, the effect of elagolix is rapidly reversible independently of the daily doses administered. ${ }^{61}$ Already from this Phase I study, it appeared that the suppressive effect of elagolix on pituitary-gonadal axis is dose dependent, and this is a remarkable property of non-peptide GnRHant. These results were confirmed by a second Phase I trial evaluating multipleascending doses in healthy premenopausal women for 21 days. $^{62}$ The study demonstrated a rapid, dose-dependent suppression of gonadotropins and estradiol after elagolix administration; the maximal estradiol suppression occurred at doses of $200 \mathrm{mg}$ twice daily or higher, and progesterone remained at anovulatory concentrations over the 21 days at doses of $100 \mathrm{mg}$ twice daily or higher. ${ }^{62}$

Clinical pharmacokinetic studies showed that elagolix is rapidly absorbed through the gastrointestinal tract following oral administration, and it achieves the peak plasma concentrations in 30-60 mins. Plasma pharmacokinetic showed a dose-dependent profile. Exposure is reduced by approximately $25 \%$ by food. Plasma half-life of elagolix across the range of studied doses changed from about 2 to $6 \mathrm{hrs}$. Repeated daily administrations result in minimal or no significant accumulation of this drug. ${ }^{61,62}$ Elagolix undergoes hepatic metabolism, mainly mediated by CYP3A4, with a $90 \%$ of excretion in the feces, and it can induce a weak inhibition of this enzyme. ${ }^{57,61}$ Less than $3 \%$ of the orally administered dose is excreted unchanged in the urine. Plasma exposure of elagolix metabolite is less than $3 \%$, and the metabolite effects are not clinically relevant. ${ }^{61}$ Renal impairment has no effect on pharmacokinetic exposures of elagolix and no dose adjustment is required in women with renal disease. On the contrary, elagolix exposure is increased by approximately 3 to 7 -fold in presence of moderate to severe hepatic impairment; therefore, attention must be used in women with hepatic disease. ${ }^{63}$

\section{Efficacy Studies For Uterine Fibroids}

The clinical trials evaluating elagolix for the treatment of symptomatic UFs assessed mainly the effect of the drug on HMB and menstrual blood loss (MBL) in premenopausal women, with safety concerns. In Tables 1 and 2 the main information concerning the therapeutic clinical trials on elagolix for UFs are reported. To date, two Phase II studies have been completed and published data are available. ${ }^{64,65}$ The Phase IIa study reported by Archer et al is a doseranging, placebo-controlled trial conducted in about 250 premenopausal women (18-49 years old) with UFs and MBL $>80 \mathrm{~mL}$ per cycle. ${ }^{64}$ They were randomized to 3month treatment with a total daily dose of elagolix alone 200-400 or $600 \mathrm{mg}$, or elagolix plus add-back therapy (200 mg twice daily plus continuous low-dose estradiol $0.5 \mathrm{mg} /$ norethindrone acetate (NETA) $0.1 \mathrm{mg}$ or elagolix $300 \mathrm{mg}$ twice daily plus estradiol $1 \mathrm{mg}$ continuously and cyclical progesterone $200 \mathrm{mg}$ ), or placebo. Elagolix 


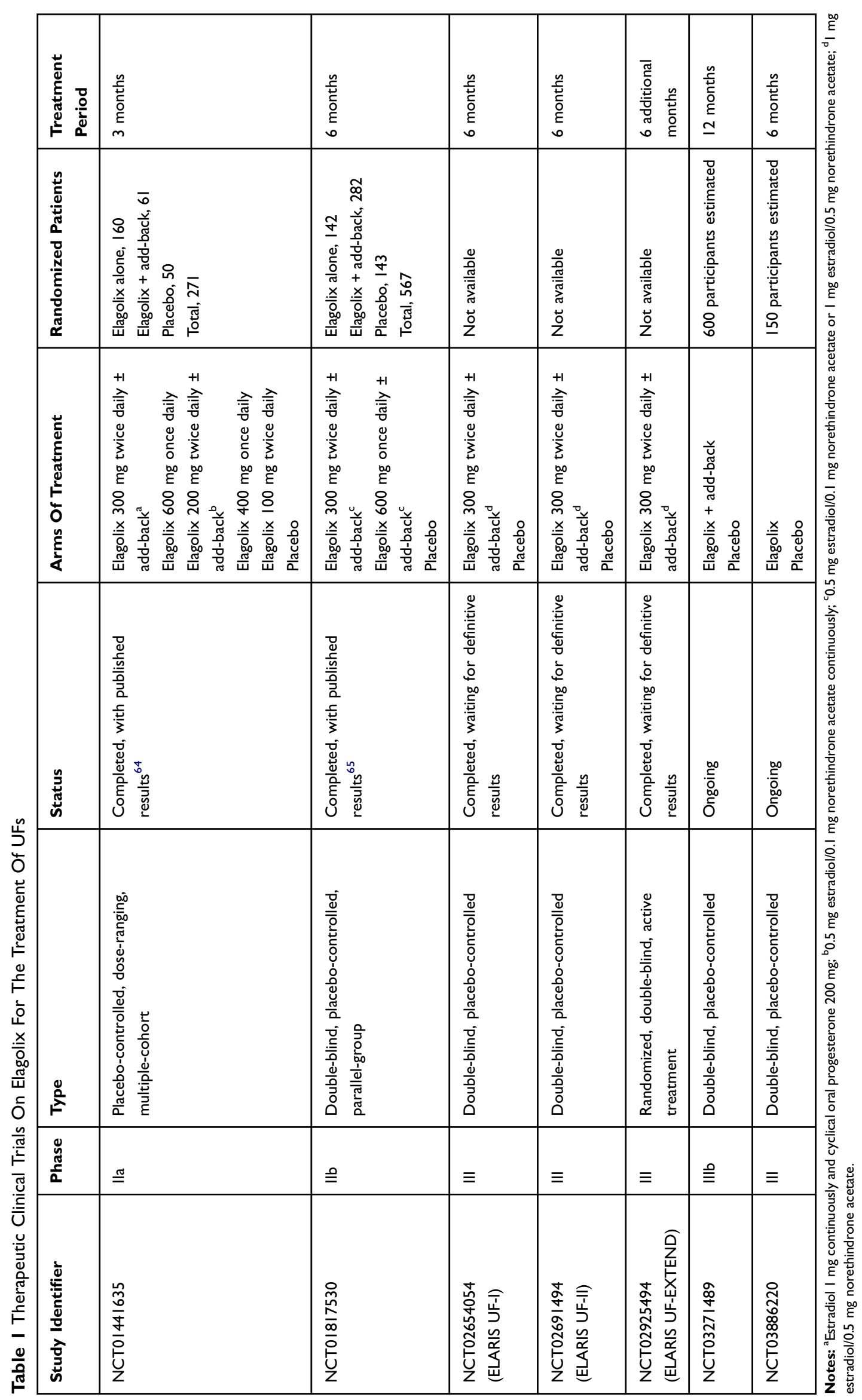


Table 2 Endpoints And Safety Assessment Of Phase II And III Clinical Trials On Elagolix For The Treatment Of Heavy Menstrual Bleeding-Associated With Uterine Fibroids

\begin{tabular}{|c|c|}
\hline Primary endpoint & - percentage of women with reduction in $\mathrm{MBL}$ to $<80 \mathrm{~mL}$ and $\geq 50 \%$ from baseline \\
\hline Secondary endpoint & $\begin{array}{l}\text { - percentage of women with amenorrhea and suppression of bleeding } \\
\text { - mean change from baseline in the number of bleeding and heavy bleeding days } \\
\text { - percentage of women who had a I g/dL or greater increase in hemoglobin concentration } \\
\text { - mean change from baseline in hemoglobin concentration } \\
\text { - mean change from baseline in Uterine Fibroid Symptom and Health Related Quality of Life Questionnaire score }\end{array}$ \\
\hline Safety assessment & $\begin{array}{l}\text { - adverse events monitoring } \\
\text { - vital signs, electrocardiogram and physical examination } \\
\text { - clinical laboratory tests (hematology, chemistry, urinalysis, lipid panel) } \\
\text { - bone mineral density assessment (dual-energy X-ray absorptiometry scans) } \\
\text { - endometrial assessment (tissue biopsy and transvaginal ultrasound) }\end{array}$ \\
\hline
\end{tabular}

Abbreviation: MBL, menstrual blood loss.

significantly improved mean percentage change in MBL from baseline to month 3 compared to placebo, achieving a dose-dependent reduction of $72-98 \%$ versus $8-41 \%$ with placebo $(\mathrm{p} \leq 0.01)$; mean percentage changes in add-back groups were $80-85 \%$, with a minor occurrence of adverse events (AEs). The reduction in MBL was associated with an increase in haemoglobin concentrations in all elagolix groups at month $3 .{ }^{64} \mathrm{~A}$ second multicentre, randomized, double-blind, placebo-controlled, Phase IIb study compared elagolix alone or with add-back therapy $(0.5 \mathrm{mg}$ estradiol/0.1 mg NETA, or $1 \mathrm{mg}$ estradiol/0.5 mg NETA) at the doses of $300 \mathrm{mg}$ twice daily or $600 \mathrm{mg}$ once daily, versus placebo, for a 6 -month treatment period. ${ }^{65}$ The primary endpoint was the reduction of $50 \%$ or higher in MBL from baseline to the final month of treatment, in women with UFs and HMB $(>80 \mathrm{~mL})$. With a sample of about 550 subjects, the trial confirmed that elagolix with or without add-back therapy is able to significantly decrease MBL: the mean percent change in MBL from baseline to final visit ranged from $71 \%$ to $93 \%$ in the $300 \mathrm{mg}$ twice daily groups compared to $24 \%$ with placebo, and from $69 \%$ to $88 \%$ in the $600 \mathrm{mg}$ once daily groups compared to $25 \%$ with placebo. As second outcomes, a significantly higher percentage of women in the elagolix groups attained amenorrhea or suppression of bleeding and improved haemoglobin concentrations compared with placebo; all elagolix groups showed a decrease in total uterine and leiomyoma volume from baseline to the final visit compared to placebo. ${ }^{65}$ There are no data in this trial about fibroid size after the stop of the treatment, and very few data are available about the recurrence of symptoms. The Phase IIa study of Archer et al reported a variable increase of fibroids volume at the third month of follow-up, after stopping active treatment. ${ }^{64}$ Two randomized, double-blind, placebo-controlled, Phase III studies were designed to assess the efficacy of a 6-month treatment with elagolix $300 \mathrm{mg}$ twice daily, with or without add-back $1 \mathrm{mg}$ estradiol/0.5 mg NETA daily, for the treatment of HMB associated with UFs (ELARIS UF-I and ELARIS UF-II). ${ }^{66,67}$ Premenopausal women aged 18 to 51 years with $\mathrm{MBL}>80 \mathrm{~mL}$ per cycle were recruited in multiple sites of United States and Canada. Preliminary results on these Phase III clinical trials were recently presented in abstract form and by official announcement by the sponsor. Elagolix treatment, in combination with low-dose add-back therapy, showed to significantly reduce HMB associated with UFs compared to placebo, with the $68.5 \%$ of women that achieved a $50 \%$ or higher reduction in MBL from baseline to final month in ELARIS UF-1 and the $76.2 \%$ of women in ELARIS UF-2. ${ }^{6,69}$ To evaluate the long-term efficacy and safety of elagolix treatment, the experimental program planned an extension study consequent to the two pivotal trials, the ELARIS UF-EXTEND, that has been completed in march 2019. In ELARIS UFEXTEND, patients from the active treatment groups in ELARIS UF-I or -II received an additional 6 months of the same treatment, while patients who had initially received placebo were randomized to either elagolix alone or elagolix plus add-back therapy. ${ }^{70}$ Preview results of the extension study are consistent with that observed in the pivotal studies, confirming the efficacy of elagolix on the control of $\mathrm{HMB}$, and no new safety signals were identified during the additional 6 months of treatment. ${ }^{71}$ Two further Phase III clinical trial are currently ongoing 
and are recruiting with the aim of evaluating the efficacy and safety of a long-term treatment (12 months) with elagolix and low-dose hormone add-back therapy compared to placebo, ${ }^{72}$ and a 6 months treatment with elagolix alone compared to placebo. ${ }^{73}$ If the definitive results on safety and efficacy of Phase III trials are confirmed as positive, it is supposed that they will sustain the regulatory submission of elagolix for the treatment of symptomatic UFs.

\section{Safety And Tolerability}

Concerning elagolix safety, more data are available from pre-marketing studies conducted in women with endometriosis. In clinical trials for the treatment of both endometriosis-associated pain and HMB related to UFs, elagolix administration was generally considered well tolerated, with most AEs being of mild to moderate severity. ${ }^{57,58}$ To assess safety, treatment-emergent AEs and serious AEs were monitored and recorded, and women participating in the clinical trials underwent vital signs, ECG and clinical laboratory parameter assessments. In endometriosis trials, where elagolix was tested without any add-back therapy, AEs were reported by $79-85 \%$ of women in the pivotal Phase III trials with a dose-related trend, versus $72-74 \%$ of placebo group, and in approximately $90 \%$ of women over the course of 12 months in the extension studies. ${ }^{59,74}$ The most common AEs, appearing in $>5 \%$ of women with incidence higher than placebo, were hot flushes and night sweats, headache, nausea, insomnia, mood swings, depressive symptoms and anxiety and arthralgia. Serious AEs most reported were abdominal pain, appendicitis and back pain. Suicidal ideation was reported in $0.2 \%$ of patients, compared to none in placebo group. ${ }^{59,74}$ Safety results from Phase II trials evaluating elagolix for UFs treatment are substantially consistent with those seen in women with endometriosis. Treatment-emergent AEs were reported in $70-87 \%$ of women from the elagolix alone groups, compared to $56-70 \%$ from the placebo groups; in the groups receiving elagolix in combination with add-back therapies, overall rates of AEs were numerically lower, ranging from $55 \%$ to $74 \% .{ }^{64,65}$ Among serious AEs, one reported in the Phase IIa study (fibroid necrosis and acute uterine haemorrhage resulting in hysterectomy) was considered possibly related to treatment. ${ }^{64}$ The most common AEs, according to endometriosis studies, were hot flushes, headache and nausea, even if the concomitant use of add-back therapy significantly reduced hot flushes appearance. ${ }^{64,65}$ All elagolix treatment groups, except the $300 \mathrm{mg}$ twice daily with
$1 \mathrm{mg}$ estradiol/0.5 mg NETA, had significant differences compared with placebo in serum lipid outcomes, affecting mainly the high-density lipoprotein cholesterol. However, all the serum lipid parameter returned to baseline in the post-treatment follow-up period. ${ }^{64,65}$ There were no other meaningful changes in laboratory parameters, except the increases in haemoglobin concentrations in elagolix groups. ${ }^{64}$ Occasional increases of aspartate or alanine aminotransferase were reported, and they spontaneously resolved also for the women who remained on treatment. ${ }^{65}$ During elagolix clinical trials, some pregnancies were reported, anyway the limited human data are insufficient to determine whether there is a risk for major congenital defects or miscarriage due to the treatment. ${ }^{58}$ Considering the hypoestrogenic effect caused by elagolix treatment, the bone health was considered with great attention during trials. A significant reduction in bone mineral density (BMD) from baseline to 6 months was shown in endometriosis studies compared with placebo: lumbar spine, femoral neck or total hip BMD decreases $>8 \%$ were observed in $1-2 \%$ of elagolix $150 \mathrm{mg}$ once daily and in $6-7 \%$ of elagolix $200 \mathrm{mg}$ twice daily treatment groups. ${ }^{59}$ After 12 months of treatment in the endometriosis extension studies, the BMD decrease was significantly greater than that observed after 6 months in active treatment groups (mean percent change from baseline in lumbar spine $-0.63 /-1.1 \%$ for the $150 \mathrm{mg}$ once-daily groups and $-3.6 /-3.9 \%$ for the $200 \mathrm{mg}$ twice-daily groups). ${ }^{74}$ Even if there are not comparative studies with leuprolide acetate, the effect on BMD reported with elagolix at each dose is lower than that reported with the GnRHa $(6.3 \%$ mean decrease in lumbar spine BMD after 12 months of leuprolide acetate alone). ${ }^{75}$ After elagolix treatment discontinuation, the dual-energy X-ray absorptiometry scans performed at the term of follow-up period (after 6 months) showed a return toward baseline of BMD values. ${ }^{74}$ The only comparative study of elagolix available, assessed the drug versus subcutaneous depot medroxyprogesterone acetate (DMPA) for the treatment of endometriosis, for 24 weeks treatment period: the study showed that both treatments with elagolix administered at $150 \mathrm{mg}$ once daily or $75 \mathrm{mg}$ twice daily, and that with DMPA were associated with a similar reduction from baseline of BMD; thus, at these doses and for 24 weeks, the impact of elagolix on bone health was considered minimal by the authors. ${ }^{76}$ In the Phase IIb study for the treatment of heavy bleeding related to UFs, safety results on bone health showed that a significant decrease in BMD compared 
with placebo (mean percent change from baseline to month 6 measured at the lumbar spine, total hip, and femoral neck) was observed in both cohorts of women in the elagolix groups without or with $0.5 \mathrm{mg}$ estradiol $/ 0.1$ mg NETA, whereas women treated with elagolix and $1 \mathrm{mg}$ estradiol/0.5 mg NETA did not show significant changes in BMD compared with placebo. ${ }^{65}$ The analysis of bone biomarkers (C-terminal collagen telopeptide and procollagen type $1 \mathrm{~N}$-terminal propeptide) performed in this study highlighted a significant increase in the groups with elagolix alone from baseline to month 6 in bone resorption and formation biomarker concentrations compared with placebo, whereas elagolix plus add-back therapy did not show significant changes. Furthermore, bone biomarker concentrations returned to baseline values at month 6 of post-treatment follow-up. ${ }^{65}$ Therefore, the addition of appropriate dose of add-back therapy at elagolix treatment seems to be able to prevent bone loss and in improving safety during long-term treatment. Data from the extension study for fibroids could give more information on safety and tolerability.

\section{Patient Acceptability}

In the published Phase II studies that evaluated elagolix treatment in women with HMB associated with UFs, as second efficacy outcomes the Uterine Fibroid Symptom and Health Related Quality of Life Questionnaire (UFSQOL) was used to assess disease-specific quality of life symptom severity scores during treatment period, compared with baseline results. ${ }^{77}$ In both studies, all elagolix groups showed significant improvement from baseline to the final visit in UFSQOL compared with placebo. ${ }^{64,65}$ The improvement in symptoms and quality of life likely related to the bleeding pattern achieved during active treatment. The occurrence of AEs may have limited patient acceptability. Nevertheless, the Phase IIa trials revealed $9 \%$ rate of discontinuation due to AEs (mainly hot flushes and headache) versus $8 \%$ in placebo group, while the Phase IIb study reported a percentage of women who discontinued the treatment for hot flushes between $0 \%$ and $4 \%$, and no discontinuation for this reason in elagolix with $1 \mathrm{mg}$ estradiol/ $0.5 \mathrm{mg}$ NETA.

\section{Conclusion}

Uterine fibromatosis constitutes a serious and widespread health problem for women of reproductive age, as well as those approaching menopause. To date, there are no efficient and structured preventive approaches to reduce the development of leiomyomas, their growth and the related symptoms. ${ }^{35}$ Surgery has still a very prominent role in the treatment of symptomatic fibroids, but especially in view of the increase in average age at the first pregnancy and the desire of fertility-sparing management, more nonsurgical options are necessary. In women who refuse surgery, the problem of the chronic drug use and the appearance of side effects arise. Therefore, the need of an efficient, safe, cheap and acceptable medical treatment for UFs is deeply required. The SPRMs obtained a great success and UPA were introduced initially for brief pre-operatory treatment and subsequently as more prolonged therapy, thanks to its effect on bleeding pattern and the slight action on fibroid volume. $^{45}$ Nevertheless, UPA received approval in Europe and Canada only, and recent advisory in terms of hepatic safety brought back a limitation of use. The GnRHa, despite their great action on UFs, are recommended for restricted treatment period, because of the hypoestrogenic side effects. ${ }^{12}$ The GnRHant could represent a new alternative for the management of this gynaecological condition. The main important characteristic of the GnRHant is the quick and dose-dependent inhibition of gonadal axis, that can lead to a reduction of AEs occurrence and severity, and to a better tolerability in comparison with the GnRHa, for the ability of the antagonists to not totally suppress estradiol levels. The studies on elagolix showed an acceptable safety and tolerability profile. The AEs reported with its use were usually mild or moderate in intensity and were consistent with the drug's mechanism of action. Furthermore, the pharmacokinetic and pharmacodynamic characteristics of elagolix permit a rapid reversibility of action after treatment discontinuation. ${ }^{57}$ This property is useful for women of reproductive age who may plan pregnancy, but also has the drawback of the symptom recurrence with the resumption of menses. Thanks to the combination of elagolix with an adequate low dose of add-back hormone therapy, there is the possibility of carrying out an effective treatment, free of severe hypoestrogenic effect and safe for bone health, which could represent a great achievement in gynecology. Optimal results seem to be obtained using the dose of $300 \mathrm{mg}$ twice daily with add-back $1 \mathrm{mg}$ estradiol/ $0.5 \mathrm{mg}$ NETA. ${ }^{65}$ The complete results of the Phase III clinical trials on elagolix for UFs are awaited to confirm these expectations and the elagolix safety for long-term therapy. Moreover, comparative studies with GnRHa or other common treatment used for symptomatic UFs would be useful to test the benefit/risk ratio superiority of elagolix, which 
so far has been supposed based on its manageability, good bioavailability, rapid onset of action and reversibility, and minor AEs profile.

\section{Abbreviations}

AEs, adverse events; BMD, bone mineral density; DMPA, depot medroxyprogesterone acetate; EMA, European Medicines Agency; ER, estrogen receptors; FSH, folliclestimulating hormone; GnRHa, gonadotropin-releasing hormone agonists; GnRHant, gonadotropin-releasing hormone antagonists; HMB, heavy menstrual bleeding; LH, luteinizing hormone; LNG-IUS, levonorgestrel-releasing intrauterine systems; MBL, menstrual blood loss; MPA, medroxyprogesterone acetate; NETA, norethindrone acetate; PR, progesterone receptors; PRAC, Pharmacovigilance Risk Assessment Committee; QoL, quality of life; SPRM, selective progesterone-receptor modulator; UFs, uterine fibroids; UFSQOL, Uterine Fibroid Symptom and Health Related Quality of Life Questionnaire; UPA, ulipristal acetate.

\section{Acknowledgments}

The authors thank Kate Jenkins for revising the English language of the manuscript. All the authors remember with affection, gratitude and esteem their colleague and friend, Dr. Marisa Orrù, who left us prematurely.

\section{Disclosure}

The authors report no conflicts of interest in this work.

\section{References}

1. Stewart EA, Cookson CL, Gandolfo RA, Schulze-Rath R. Epidemiology of uterine fibroids: a systematic review. BJOG. 2017;124(10):1501-1512. doi:10.1111/1471-0528.14640

2. Bulun S. Uterine fibroids. $N$ Engl J Med. 2013;369(14):1344-1365. doi:10.1056/NEJMra1209993

3. Catherino WH, Parrott E, Segars J. Proceedings from the National Institute of Child Health and Human Development conference on the Uterine Fibroid Research Update Workshop. Fertil Steril. 2011;95 (1):9-12. doi:10.1016/j.fertnstert.2010.08.049

4. Ben-Nagi J, Miell J, Mavrelos D, Naftalin J, Lee C, Jurkovic D. Endometrial implantation factors in women with submucous uterine fibroids. Reprod Biomed Online. 2010;21(5):610-615. doi:10.1016/j. rbmo.2010.06.039

5. Sunkara SK, Khairy M, El-Toukhy T, Khalaf Y, Coomarasamy A. The effect of intramural fibroids without uterine cavity involvement on the outcome of IVF treatment: a systematic review and meta-analysis. Hum Reprod. 2010;25(2):418-429. doi:10.1093/humrep/dep396

6. Zepiridis LI, Grimbizis GF, Tarlatzis BC. Infertility and uterine fibroids. Best Pract Res Clin Obstet Gynaecol. 2016;34:66-73. doi:10.1016/j.bpobgyn.2015.12.001

7. Parazzini F, Tozzi L, Bianchi S. Pregnancy outcome and uterine fibroids. Best Pract Res Clin Obstet Gynaecol. 2016;34:74-84. doi:10.1016/j.bpobgyn.2015.11.017
8. Hervé F, Katty A, Isabelle Q, Céline S. Impact of uterine fibroids on quality of life: a national cross-sectional survey. Eur J Obstet Gynecol Reprod Biol. 2018;229:32-37. doi:10.1016/j.ejogrb.2018.07.032

9. Downes E, Sikirica V, Gilabert-Estelles J, et al. The burden of uterine fibroids in five European countries. Eur J Obstet Gynecol Reprod Biol. 2010;152(1):96-102. doi:10.1016/j.ejogrb.2010.05.012

10. Soliman AM, Margolis MK, Castelli-Haley J, Fuldeore MJ, Owens CD, Coyne KS. Impact of uterine fibroid symptoms on health-related quality of life of US women: evidence from a cross-sectional survey. Curr Med Res Opin. 2017;33(11):1971-1978. doi:10.1080/03007995.2017.1372107

11. Cardozo ER, Clark AD, Banks NK, Henne MB, Stegmann BJ, Segars JH. The estimated annual cost of uterine leiomyomata in the United States. $A m$ J Obstet Gynecol. 2012;206(3):211.e1-9. doi:10.1016/j.ajog.2011.12.002

12. Stewart EA. Clinical practice. Uterine fibroids. $N$ Engl $J$ Med. 2015;372(17):1646-1655. doi:10.1056/NEJMcp1411029

13. Pérez-López FR, Ornat L, Ceausu I, et al. EMAS position statement: management of uterine fibroids. Maturitas. 2014;79(1):106-116. doi:10.1016/j.maturitas.2014.06.002

14. Mais V, Ajossa S, Guerriero S, Mascia M, Solla E, Melis GB. Laparoscopic versus abdominal myomectomy: a prospective, randomized trial to evaluate benefits in early outcome. Am J Obstet Gynecol. 1996;174(2):654-658. doi:10.1016/s0002-9378(96)70445-3

15. Jin C, Hu Y, Chen XC, et al. Laparoscopic versus open myomectomy - a meta-analysis of randomized controlled trials. Eur J Obstet Gynecol Reprod Biol. 2009;145(1):14-21. doi:10.1016/j.ejogrb.2009.03.009

16. US Food and Drug Administration. Laparoscopic uterine power morcellation in hysterectomy and myomectomy: FDA safety communication. 2014. Available from: https://wayback.archive-it.org/ 7993/20170404182209/https:/www.fda.gov/MedicalDevices/Safety/ AlertsandNotices/ucm424443.htm. Accessed March 31, 2019.

17. Siedhoff MT, Cohen SL. Tissue extraction techniques for leiomyomas and uteri during minimally invasive surgery. Obstet Gynecol. 2017;130(6):1251-1260. doi:10.1097/AOG.0000000000002334

18. Friedman JA, Wong JMK, Chaudhari A, Tsai S, Milad MP. Hysteroscopic myomectomy: a comparison of techniques and review of current evidence in the management of abnormal uterine bleeding. Curr Opin Obstet Gynecol. 2018;30(4):243-251. doi:10.1097/ GCO.0000000000000475

19. Borah BJ, Nicholson WK, Bradley L, Stewart EA. The impact of uterine leiomyomas: a national survey of affected women. Am J Obstet Gynecol. 2013;209(4):319.e1-319.e20. doi:10.1016/j.ajog.2013.07.017

20. Donnez J, Dolmans MM. Uterine fibroid management: from the present to the future. Hum Reprod Update. 2016;22(6):665-686. doi:10.1093/humupd/dmw023

21. Lee BB, Yu SP. Radiofrequency ablation of uterine fibroids: a review. Curr Obstet Gynecol Rep. 2016;5(4):318-324.

22. Hudgens J, Johns DA, Lukes AS, Forstein DA, Delvadia D. 12month outcomes of the US patient cohort in the SONATA pivotal IDE trial of transcervical ablation of uterine fibroids. Int $J$ Womens Health. 2019;(11):387-394. doi:10.2147/IJWH.S201912

23. Linder D, Gartler SM. Glucose-6-phosphate dehydrogenase mosaicism: utilization as a cell marker in the study of leiomyomas. Science. 1965;150(3692):67-69. doi:10.1126/science.150.3692.67

24. Ono M, Qiang W, Serna VA, et al. Role of stem cells in human uterine leiomyoma growth. PLoS One. 2012;7(5):e36935. doi:10.1371/journal.pone. 0036935

25. Marsh EE, Bulun SE. Steroid hormones and leiomyomas. Obstet Gynecol Clin North Am. 2006;33(1):59-67. doi:10.1016/j.ogc.2005.12.001

26. Ishikawa H, Ishi K, Serna VA, Kakazu R, Bulun SE, Kurita T. Progesterone is essential for maintenance and growth of uterine leiomyoma. Endocrinology. 2010;151(6):2433-2442. doi:10.1210/en.20091225

27. Sumitani H, Shozu M, Segawa T, et al. In situ estrogen synthesized by aromatase P450 in uterine leiomyoma cells promotes cell growth probably via an autocrine/intracrine mechanism. Endocrinology. 2000;141(10):3852-3861. doi:10.1210/endo.141.10.7719 
28. Parsanezhad ME, Azmoon M, Alborzi S, et al. A randomized, controlled clinical trial comparing the effects of aromatase inhibitor (letrozole) and gonadotropin releasing hormone agonist (triptorelin) on uterine leiomyoma volume and hormonal status. Fertil Steril. 2010;93(1):192-198. doi:10.1016/j.fertnstert.2008.09.064

29. Duhan N, Madaan S, Sen J. Role of the aromatase inhibitor letrozole in the management of uterine leiomyomas in premenopausal women. Eur J Obstet Gynecol Reprod Biol. 2013;171(2):329-332. doi:10.1016/j.ejogrb.2013.09.010

30. Song H, Lu D, Navaratnam K, Shi G. Aromatase inhibitors for uterine fibroids. Cochrane Database Syst Rev. 2013;10:CD009505.

31. Qin J, Yang T, Kong F, Zhou Q. Oral contraceptive use and uterine leiomyoma risk: a meta-analysis based on cohort and case-control studies. Arch Gynecol Obstet. 2013;288(1):139-148. doi:10.1007/ s00404-013-2797-9

32. Jiang W, Shen Q, Chen M, et al. Levonorgestrel releasing intrauterine system use in premenopausal women with symptomatic uterine leiomyoma: a systematic review. Steroids. 2014;86:69-78. doi:10.1016/j. steroids.2014.05.002

33. Orbo A, Arnes M, Pettersen I, Larsen K, Hanssen K, Moe B. Downregulated progesterone receptor A and B coinciding with successful treatment of endometrial hyperplasia by the levonorgestrel impregnated intrauterine system. Acta Obstet Gynecol Scand. 2010;89(11):1438-1446. doi:10.3109/00016349.2010.512068

34. Ciebiera M, Łukaszuk K, Męczekalski B, et al. Alternative oral agents in prophylaxis and therapy of uterine fibroids-an up-to-date review. Int J Mol Sci. 2017;18(12):pii:E2586. doi:10.3390/ijms18122586

35. Bartels CB, Cayton KC, Chuong FS, et al. An evidence-based approach to the medical management of fibroids: a systematic review. Clin Obstet Gynecol. 2016;59(1):30-52. doi:10.1097/GRF.0000000000000171

36. Letteri GS, Coddington CC, Winkel CA, Shawker TH, Loriaux DL, Collins RL. Efficacy of gonadotropin releasing hormone agonist in the treatment of uterine leiomyomata: long-term follow-up. Fertil Steril. 1989;51(6):951-956. doi:10.1016/s0015-0282(16)60724-0

37. Paoletti AM, Serra GG, Cagnacci A, et al. Spontaneous reversibility of bone loss induced by gonadotropin-releasing hormone analog treatment. Fertil Steril. 1996;65(4):707-710.

38. Lethaby A, Vollenhoven B, Sowter M. Pre-operative GnRH analogue therapy before hysterectomy or myomectomy for uterine fibroids. Cochrane Database Syst Rev. 2001;2:CD000547.

39. Moroni RM, Martins WP, Ferriani RA, et al. Add-back therapy with GnRH analogues for uterine fibroids. Cochrane Database Syst Rev. 2015;3:CD010854.

40. Bouchard P, Chabbert-Buffet N, Fauser BC. Selective progesterone receptor modulators in reproductive medicine: pharmacology, clinical efficacy and safety. Fertil Steril. 2011;96(5):1175-1189. doi:10.1016/ j.fertnstert.2011.08.021

41. Chabbert-Buffet N, Meduri G, Bouchard P, Spitz IM. Selective progesterone receptor modulators and progesterone antagonists: mechanisms of action and clinical applications. Hum Reprod Update. 2005;11(3):293-307. doi:10.1093/humupd/dmi002

42. Donnez J, Tatarchuk TF, Bouchard P, et al. Ulipristal acetate versus placebo for fibroid treatment before surgery. $N$ Engl J Med. 2012;366:409-420. doi:10.1056/NEJMoa1103182

43. Donnez J, Tomaszewski J, Vazquez F, et al. Ulipristal acetate versus leuprolide acetate for uterine fibroids. $N$ Engl J Med. 2012;366 (5):421-432. doi:10.1056/NEJMoa1103180

44. Biglia N, Carinelli S, Maiorana A, D’Alonzo M, Lo Monte G, Marci R. Ulipristal acetate: a novel pharmacological approach for the treatment of uterine fibroids. Drug Des Dev Ther. 2014;8:285-292.

45. Donnez J, Donnez O, Matule D, et al. Long-term medical management of uterine fibroids with ulipristal acetate. Fertil Steril. 2016;105 (1):165-173. doi:10.1016/j.fertnstert.2015.09.032

46. Fauser BC, Donnez J, Bouchard P, et al. Safety after extended repeated use of ulipristal acetate for uterine fibroids. PLoS One. 2017;12(3):e0173523. doi:10.1371/journal.pone.0173523
47. European Medicine Agency. Esmya: new measures to minimise risk of rare but serious liver injury. EMA/355940/2018. June 1, 2018. Available from: https://www.ema.europa.eu/en/documents/pressrelease/esmya-new-measures-minimise-risk-rare-serious-liver-injury_ en-0.pdf. Accessed April 13, 2019.

48. Melis GB, Neri M, Piras B, et al. Vilaprisan for treating uterine fibroids. Expert Opin Investig Drugs. 2018;27(5):497-505. doi:10.1080/13543784.2018.1471134

49. Felberbaum RE, Küpker W, Krapp M, Gehl B, Ludwig M, Diedrich K. Preoperative reduction of uterine fibroids in only 16 days by administration of a gonadotrophin-releasing hormone antagonist (Cetrotide). Reprod Biomed Online. 2001;3(1):14-18. doi:10.1016/ s1472-6483(10)61957-8

50. Flierman PA, Oberyé JJ, van der Hulst VP, de Blok S. Rapid reduction of leiomyoma volume during treatment with the $\mathrm{GnRH}$ antagonist ganirelix. BJOG. 2005;112(5):638-642. doi:10.1111/j.1471-0528.2004.00504.x

51. Tan O, Bukulmez O. Biochemistry, molecular biology and cell biology of gonadotropin-releasing hormone antagonists. Curr Opin Obstet Gynecol. 2011;23(4):238-244. doi:10.1097/GCO.0b013e328348a3ce

52. Tukun FL, Olberg DE, Riss PJ, Haraldsen I, Kaass A, Klaveness J. Recent development of non-peptide GnRH antagonists. Molecules. 2017;22(12):E2188. doi:10.3390/molecules22122188

53. Markham A. Relugolix: first Global Approval. Drugs. 2019;79 (6):675-679. doi:10.1007/s40265-019-01105-0

54. Efficacy and safety of OBE2109 in subjects with heavy menstrual bleeding associated with uterine fibroids (PRIMROSE 1). Available from: https:/clinicaltrials.gov/ct2/show/NCT03070899?view=record. ClinicalTrials.gov Identifier: NCT03070899. Accessed April 14, 2019.

55. Efficacy and safety of OBE2109 in subjects with heavy menstrual bleeding associated with uterine fibroids (PRIMROSE 2). Available from: https:/clinicaltrials.gov/ct2/show/NCT03070951?view=record. ClinicalTrials.gov Identifier: NCT03070951. Accessed April 14, 2019.

56. A study to assess the efficacy and safety of OBE2109 in subjects with endometriosis (EDELWEISS). Available from: https://clinicaltrials. gov/ct2/show/NCT02778399?view=record. ClinicalTrials.gov Identifier: NCT02778399. Accessed April 14, 2019.

57. Lamb YN. Elagolix: first global approval. Drugs. 2018;78(14):15011508. doi:10.1007/s40265-018-0977-4

58. Melis GB, Neri M, Corda V, et al. Overview of elagolix for the treatment of endometriosis. Expert Opin Drug Metab Toxicol. 2016;12(5):581-588. doi:10.1517/17425255.2016.1171316

59. Taylor HS, Giudice LC, Lessey BA, et al. Treatment of endometriosis-associated pain with elagolix, an oral GnRH antagonist. $N$ Engl J Med. 2017;377(1):28-40. doi:10.1056/NEJMoa1700089

60. Chen C, Wu D, Guo Z, et al. Discovery of sodium R-(+)-4-\{2-[5-(2fluoro-3-methoxyphenyl)-3-(2-fluoro-6-[trifluoromethyl]benzyl)-4-meth yl-2,6-dioxo-3,6-dihydro-2H-pyrimidin-1-yl]-1-phenylethylamino butyrate (elagolix), a potent and orally available nonpeptide antagonist of the human gonadotropin-releasing hormone receptor. J Med Chem. 2008;51 (23):7478-7485. doi:10.1021/jm8006454

61. Struthers RS, Nicholls AJ, Grundy J, et al. Suppression of gonadotropins and estradiol in premenopausal women by oral administration of the nonpeptide gonadotropin releasing hormone antagonist elagolix. J Clin Endocrinol Metab. 2009;94(2):545-551. doi:10.1210/ jc.2008-1695

62. Ng J, Chwalisz K, Carter DC, Klein CE. Dose-dependent suppression of gonadotropins and ovarian hormones by elagolix in healthy premenopausal women. J Clin Endocrinol Metab. 2017;102(5):16831691. doi:10.1210/jc.2016-3845

63. Ng J, Duan WR, Marbury T, Schmidt JM, Klein CE. Elagolix pharmacokinetic profiles in women with renal or hepatic impairment. Clin Pharmacol Drug Dev. 2018;1-9.

64. Archer DF, Stewart EA, Jain RI, et al. Elagolix for the management of heavy menstrual bleeding associated with uterine fibroids: results from a phase 2a proof-of-concept study. Fertil Steril. 2017;108 (1):152-160.e4. doi:10.1016/j.fertnstert.2017.05.006 
65. Carr BR, Stewart EA, Archer DF, et al. Elagolix alone or with addback therapy in women with heavy menstrual bleeding and uterine leiomyomas: a randomized controlled trial. Obstet Gynecol. 2018;132 (5):1252-1264. doi:10.1097/AOG.0000000000002933

66. Efficacy and safety of elagolix in combination with estradiol/norethindrone acetate for the management of heavy menstrual bleeding associated with uterine fibroids in premenopausal women. Available from: https:// clinicaltrials.gov/ct2/show/NCT02654054?view=record. ClinicalTrials. gov Identifier: NCT02654054. Accessed April 22, 2019.

67. Efficacy and safety of elagolix in combination with estradiol/norethindrone acetate for the management of heavy menstrual bleeding associated with uterine fibroids in premenopausal women (Replicate study). Available from: https://clinicaltrials.gov/ct2/show/record/ NCT02691494?view=record. ClinicalTrials.gov Identifier: NCT02691 494. Accessed April 22, 2019.

68. Carr B, Bradley L, Al-Hendy A, et al. Elagolix treatment in women with heavy menstrual bleeding-associated with uterine fibroids: efficacy and safety results from two double-blind, randomized, placebocontrolled Phase 3 studies. J Minim Invasive Gynecol. 2018;25(7): S88. doi:10.1016/j.jmig.2018.09.178

69. AbbVie News Center. AbbVie presents positive phase 3 data demonstrating investigational elagolix reduces heavy menstrual bleeding in women with uterine fibroids at 2018 AAGL global congress. November 14, 2018. Available from: https://news.abbvie.com/news/ press-releases/abbvie-presents-positive-phase-3-data-demonstratinginvestigational-elagolix-reduces-heavy-menstrual-bleeding-inwomen-with-uterine-fibroids-at-2018-aagl-global-congress.htm. Accessed April 22, 2019.

70. An extension study to evaluate the efficacy and safety of elagolix in premenopausal women with heavy menstrual bleeding associated with uterine fibroids. ClinicalTrials.gov Identifier: NCT02925494. Available from: https://clinicaltrials.gov/ct2/show/NCT02925494? view=record. Accessed April 22, 2019.
71. AbbVie News Center. AbbVie announces positive topline results from Phase 3 extension study evaluating investigational elagolix in women with uterine fibroids. August 22, 2018. Available from: https://news.abbvie. com/news/press-releases/abbvie-announces-positive-topline-resultsfrom-phase-3-extension-study-evaluating-investigational-elagolix-inwomen-with-uterine-fibroids.htm. Accessed April 22, 2019

72. Long-term safety and efficacy of elagolix in combination with estradiol/norethindrone acetate for the management of heavy menstrual bleeding associated with uterine fibroids in premenopausal women. Available from: https://clinicaltrials.gov/ct2/show/NCT03271489? view=record. ClinicalTrials.gov Identifier: NCT03271489. Accessed April 22, 2019.

73. A study to evaluate the safety and efficacy of elagolix for the management of heavy menstrual bleeding associated with uterine fibroids in premenopausal women. Available from: https://clinical trials.gov/ct2/show/NCT03886220?view=record. ClinicalTrials.gov Identifier: NCT03886220. Accessed April 22, 2019.

74. Surrey E, Taylor HS, Giudice L, et al. Long-term outcomes of elagolix in women with endometriosis: results from two extension studies. Obstet Gynecol. 2018;132(1):147-160. doi:10.1097/AOG.0000000000002675

75. Hornstein MD, Surrey ES, Weisberg GW, Casino LA; Lupron AddBack Study Group. Leuprolide acetate depot and hormonal add-back in endometriosis: a 12-month study. Obstet Gynecol. 1998;91(1):1624. doi:10.1016/s0029-7844(97)00620-0

76. Carr B, Dmowski WP, O'Brien C, et al. Elagolix, an oral GnRH antagonist, versus subcutaneous depot medroxyprogesterone acetate for the treatment of endometriosis: effects on bone mineral density. Reprod Sci. 2014;21(11):1341-1351. doi:10.1177/1933719114549848

77. Coyne KS, Soliman AM, Margolis MK, Thompson CL, Chwalisz K. Validation of the 4 week recall version of the Uterine Fibroid Symptom and Health-related Quality of Life (UFS-QOL) Questionnaire. Curr Med Res Opin. 2017;33(2):193-200. doi:10.1080/03007995.2016.1248382

\section{Publish your work in this journal}

The International Journal of Women's Health is an international, peerreviewed open-access journal publishing original research, reports, editorials, reviews and commentaries on all aspects of women's healthcare including gynecology, obstetrics, and breast cancer. The manuscript management system is completely online and includes a very quick and fair peer-review system, which is all easy to use. Visit http://www.dovepress.com/testimonials.php to read real quotes from published authors. 